\title{
Real-Time Electron Dynamics with Exact-Exchange Time-Dependent Density-Functional Theory
}

\author{
H. O. Wijewardane* and C. A. Ullrich \\ Department of Physics and Astronomy, University of Missouri, Columbia, Missouri 65211, USA
}

(Received 28 August 2007; published 7 February 2008)

\begin{abstract}
The exact exchange potential in time-dependent density-functional theory is defined as an orbital functional through the time-dependent optimized effective potential (TDOEP) method. We numerically solve the TDOEP integral equation for the real-time nonlinear intersubband electron dynamics in a semiconductor quantum well with two occupied subbands. It is found that memory effects become significant in the vicinity of intersubband resonances.
\end{abstract}

DOI: 10.1103/PhysRevLett.100.056404

Time-dependent density-functional theory (TDDFT) [1] has been very successful in describing electronic excitations in large molecules [2] using simple time-dependent exchange-correlation (XC) functionals such as the adiabatic local-density approximation (ALDA). However, neither the ALDA nor the adiabatic gradient-corrected XC functionals capture multiple or charge-transfer excitations [3,4] or excitons [5]. These difficulties are not due to intrinsic deficiencies of TDDFT, but indicate the need for better functionals: for multiple and charge-transfer excitations one must abandon the adiabatic approximation, and excitons in solids require long-range $\left(1 / q^{2}\right) \mathrm{XC}$ kernels. Moreover, for strong-field multiple ionization it was suggested that the $\mathrm{XC}$ potential should change discontinuously with the number of electrons $N$ [6].

Several promising ideas for new time-dependent XC functionals have been explored, such as current-TDDFT (TDCDFT) $[7,8]$ and many-body Green's function techniques [5]. TDCDFT is well suited to describe polarizability and collective excitations in extended systems $[9,10]$, but introduces spurious dissipation in finite systems [11]. The many-body techniques of Ref. [5] give excellent optical spectra in insulators, but cannot be easily extended into the nonlinear or the real-time domain.

The advantages of orbital-dependent functionals have long been recognized in static DFT [12]. Local XC potentials associated with orbital-dependent XC energies (e.g., exact exchange) are constructed with the optimized effective potential (OEP) method [13] or the simplified but nearly as accurate Krieger-Li-Iafrate (KLI) scheme [14]. The resulting XC potentials are self-interaction free, have the correct $-1 / r$ asymptotics for finite systems, exhibit discontinuities upon change of $N$, and generally produce high-quality orbitals, eigenvalues, and band structures [12].

This Letter deals with the time-dependent optimized effective potential (TDOEP) [15,16], which generalizes the static OEP method, carrying over the desirable properties mentioned above and introducing new features that are unique to the dynamical case. The TDOEP integral equation for the local XC potential $V_{\mathrm{xc} \sigma}$ is given by [15]
PACS numbers: 71.15.Mb, 31.15.ee, 71.45.Gm

$$
\begin{aligned}
0= & i \sum_{j=1}^{N \sigma} \int_{-\infty}^{t} d t^{\prime} \int d^{3} r^{\prime}\left[V_{\mathrm{xc} \sigma}\left(\mathbf{r}^{\prime}, t^{\prime}\right)-u_{\mathrm{xcj} \sigma}\left(\mathbf{r}^{\prime}, t^{\prime}\right)\right] \\
& \times \sum_{k=1}^{\infty} \phi_{k \sigma}\left(\mathbf{r}^{\prime}, t^{\prime}\right) \phi_{k \sigma}^{*}(\mathbf{r}, t) \phi_{j \sigma}^{*}\left(\mathbf{r}^{\prime}, t^{\prime}\right) \phi_{j \sigma}(\mathbf{r}, t)+\text { c.c. }
\end{aligned}
$$

The $\phi_{j \sigma}$ are time-dependent Kohn-Sham orbitals, $u_{\mathrm{xc} j \sigma}(\mathbf{r}, t)=\left[\phi_{j \sigma}^{*}(\mathbf{r}, t)\right]^{-1} \delta A_{\mathrm{xc}} / \delta \phi_{j \sigma}(\mathbf{r}, t)$, and $A_{\mathrm{xc}}$ is an orbital-dependent $\mathrm{XC}$ action functional (which is rigorously defined on a Keldysh contour [16]). We formally include spin $\sigma$, but consider only nonmagnetic systems.

Linearization of the TDOEP leads to a frequencydependent XC kernel [17]. This frequency dependence was found to play only a minor role in the optical absorption of insulators [18] and dynamic polarizabilities of atoms [19]. Until now there have been no applications of the full, real-time TDOEP, only of the time-dependent KLI (TDKLI) approximation [15]. An earlier unsuccessful attempt to solve Eq. (1) was plagued by numerical instabilities [20]. In this Letter, we present an algorithm for stable numerical solutions of the exact-exchange TDOEP and apply it to nonlinear electron dynamics in quantum wells.

Our goal is to explore the significance of memory effects in TDOEP. Memory-dependent XC potentials in TDCDFT cause elastic and dissipative effects in the electron dynamics [8]. In particular, the adiabatic approximation was shown to break down in the limit of large, rapid deformations [21]. Here, we study the so far unresolved question of the importance of memory in exact-exchange TDDFT in different dynamical regimes. To isolate the effects of memory in a clear-cut way, we will compare the full TDOEP with an adiabatic approximation (AOEP).

Adiabatic TDOEP scheme.-Under the implicit assumption that a system evolves so slowly that it always remains close to the ground state of a given time-dependent potential, adiabatic XC functionals have no memory and only depend on the instantaneous density or orbitals. While this is straightforward in ALDA and TDKLI, the AOEP is more complicated since the static OEP $[13,14]$ also depends on the energy eigenvalues, whose meaning in the dynamical regime is not obvious. 
We define the AOEP as that static OEP potential whose associated ground-state density equals the instantaneous $n(t)$. This requires two steps at each $t$ : First, find that static Kohn-Sham potential $V_{\mathrm{KS}}^{t}$ which produces $n(t)$ as its selfconsistent ground-state density and whose $\mathrm{XC}$ part is the static OEP. Since the external part of $V_{\mathrm{KS}}^{t}$ is in general different from the actual time-dependent external potential, and $a$ priori unknown, $V_{\mathrm{KS}}^{t}$ must be found by numerical inversion [22]. Next, plug the resulting complete set of orbitals $\phi_{j \sigma}^{t}(\mathbf{r})$ and eigenvalues $\epsilon_{j \sigma}^{t}$ into the static OEP $[13,14]$. The so-obtained AOEP potential at time $t$ will here be used self-consistently during time propagation. It then becomes identical with TDOEP in the static limit where the system remains in its ground state, and both reduce to the static OEP.

TDOEP for quantum wells. - We consider conduction electrons in $n$-doped semiconductor quantum wells in effective-mass approximation [8,9], confined along $z$. The ground-state envelope function for the $j$ th subband $\phi_{j \sigma}^{0}(z)$ follows from a one-dimensional Kohn-Sham equation, with density $n(z)=\sum_{j}^{o c c}\left|\phi_{j \sigma}^{0}(z)\right|^{2}\left(\epsilon_{F}-\epsilon_{j \sigma}\right) / \pi$ and subband and Fermi energy levels $\epsilon_{j \sigma}$ and $\epsilon_{F}$. To describe intersubband dynamics preserving the translational symmetry in the quantum well plane (ignoring disorder and phonons), we propagate the subband envelope functions using the time-dependent Kohn-Sham equation

$$
\begin{aligned}
i \frac{\partial}{\partial t} \phi_{j \sigma}(z, t)= & {\left[-\frac{1}{2} \frac{\partial^{2}}{\partial z^{2}}+V_{\mathrm{dr}}(z, t)+V_{\mathrm{conf}}(z)+V_{H}(z, t)\right.} \\
& \left.+V_{x \sigma}(z, t)\right] \phi_{j \sigma}(z, t),
\end{aligned}
$$

with initial condition $\phi_{j \sigma}\left(z, t_{0}\right)=\phi_{j \sigma}^{0}(z)$. Here, $V_{\mathrm{dr}}$ is a time-dependent driving field (see below), $V_{\text {conf }}(z)$ and $V_{H}$ are the confining square well and the Hartree potential.

Assuming that the system is in its ground state for $t<t_{0}$, one obtains the following TDOEP equation:

$$
\begin{aligned}
0= & i \sum_{j=1}^{N \sigma}\left(k_{F}^{j \sigma}\right)^{2} \int_{t_{0}}^{t} d t^{\prime} \int d z^{\prime}\left[V_{x \sigma}\left(z^{\prime}, t^{\prime}\right)-u_{x j \sigma}\left(z^{\prime}, t^{\prime}\right)\right] \\
& \times \sum_{k \neq j} \phi_{k \sigma}\left(z^{\prime}, t^{\prime}\right) \phi_{k \sigma}^{*}(z, t) \phi_{j \sigma}^{*}\left(z^{\prime}, t^{\prime}\right) \phi_{j \sigma}(z, t)+\text { c.c. } \\
& +\sum_{j=1}^{N \sigma}\left(k_{F}^{j \sigma}\right)^{2} \int d z^{\prime}\left[V_{x \sigma}^{0}\left(z^{\prime}\right)-u_{x j \sigma}^{0}\left(z^{\prime}\right)\right] \\
& \times \sum_{k \neq j} \phi_{k \sigma}^{*}(z, t) \frac{\phi_{j \sigma}^{0 *}\left(z^{\prime}\right) \phi_{k \sigma}^{0}\left(z^{\prime}\right)}{\epsilon_{j \sigma}-\epsilon_{k \sigma}} \phi_{j \sigma}(z, t)+\text { c.c. }
\end{aligned}
$$

where $\left(k_{F}^{j \sigma}\right)^{2}=2\left(\epsilon_{F}-\epsilon_{j \sigma}\right), u_{x j \sigma}$ for quantum wells has been given by Reboredo and Proetto [23], and $V_{x \sigma}^{0}, u_{x j \sigma}^{0}$, and $\phi_{j \sigma}^{0}$ follow from static OEP. The second term in Eq. (3) comes from $\phi_{j \sigma}(z, t)=\phi_{j \sigma}^{0}(z) e^{-i \epsilon_{j} t}$ for $t<t_{0}$.

Numerical algorithm. - Our numerical TDOEP approach uses a uniform spatial grid along $z$ and a time discretization in uniform steps $\Delta t$, from $t_{0}$ up until some final time $T$. Let us first consider two separate problems. (i) Assuming that a $V_{x \sigma}^{\prime}(z, t)$ is explicitly given for $t_{0} \leq$ $t<T$, Eq. (2) is easily propagated with standard CrankNicholson and predictor-corrector schemes. This yields the orbitals $\phi_{j \sigma}^{\prime}(z, t)$. (ii) In turn, assuming some $\phi_{j \sigma}^{\prime \prime}(z, t)$ 's to be given for $t_{0} \leq t<T$, Eq. (3) can be solved by discretizing the spatial and time integrals (e.g., with the trapezoidal rule), which leads to a linear equation determining $V_{x \sigma}^{\prime \prime}(z, t)$.

The full TDOEP scheme requires the simultaneous solution of Eqs. (2) and (3) over the interval $\left[t_{0}, T\right]$. We achieve this using a straightforward iterative loop, taking the orbitals calculated in step (i) as input to step (ii), and then feeding the resulting XC potential back as input to step (i). Self-consistency is reached if $\phi_{j \sigma}^{\prime \prime}=\phi_{j \sigma}^{\prime}$ and $V_{x \sigma}^{\prime \prime}=V_{x \sigma}^{\prime}$. The loop is initialized with the TDKLI approximation for $V_{x \sigma}^{\prime}$ in the first iteration step.

Let us define $G_{l}=\int_{t_{0}}^{T} d t\left|d_{l}(t)-d_{l-1}(t)\right| / \int_{t_{0}}^{T} d t\left|d_{l}(t)\right|$ to monitor convergence, where $d_{l}(t)=\int z n_{l}(z, t) d z$ is the dipole moment at the $l$ th iteration. Our algorithm is stable and rapidly convergent, as we will show below.

The instability of the step-by-step algorithm of Ref. [20] ultimately comes from the fact that the integrand of Eq. (1) vanishes at the upper limit $t=t^{\prime}$, so that $V_{x \sigma}\left(t^{\prime}\right)$ is only determined for $t^{\prime}<t$. In our iterative scheme, this indeterminacy of the XC potential affects only the final point $T$ and is therefore harmless.

Results and discussion. - We consider a 40-nm square GaAs $/ \mathrm{Al}_{0.3} \mathrm{Ga}_{0.7}$ As quantum well with conduction band effective mass $m^{*}=0.067 m$ and charge $e^{*}=e / \sqrt{13}$, and with an electronic density $N_{s}=2.2 \times 10^{11} \mathrm{~cm}^{-2}$ such that the two lowest subbands $(j=1,2)$ are occupied.

We first discuss free charge-density oscillations. The initial state is calculated in the presence of a $0.01 \mathrm{mV} / \mathrm{nm}$ static electric field. At $t_{0}=0$, the field is abruptly switched off, which puts the electrons in an excited state and triggers collective charge-density oscillations. Figure 1 shows that the convergence index $G$ of our numerical algorithm drops down to $10^{-9}$ after only 20 iterations. Convergence was similar in all TDOEP calculations. We found that the zero-force theorem was always satisfied to within the limits of numerical resolution, even for TDKLI (despite recent reports to the contrary in metal clusters [24]). The strong quantum well confinement seems to help enforce the zero-force theorem in TDKLI.

Figure 1 shows the dipole moment $d(t)$ obtained with TDOEP, AOEP, and TDKLI, as well as the TDOEP dipole power spectrum (the others are very similar). The dynamics is mainly determined by the $1 \rightarrow 2$ and $2 \rightarrow 3$ intersubband plasmons; the former dominates since $90 \%$ of the electrons sit in the first subband at the given $N_{s}$. Higher plasmons $(1 \rightarrow 4,2 \rightarrow 5)$ are orders of magnitude weaker. The differences between the methods are minor: the TDOEP oscillations are slightly faster, TDKLI is slowest, and AOEP in between, but much closer to TDKLI. The first plasmon frequency $\omega_{12}$ is $10.4 \mathrm{meV}$ in TDOEP and 

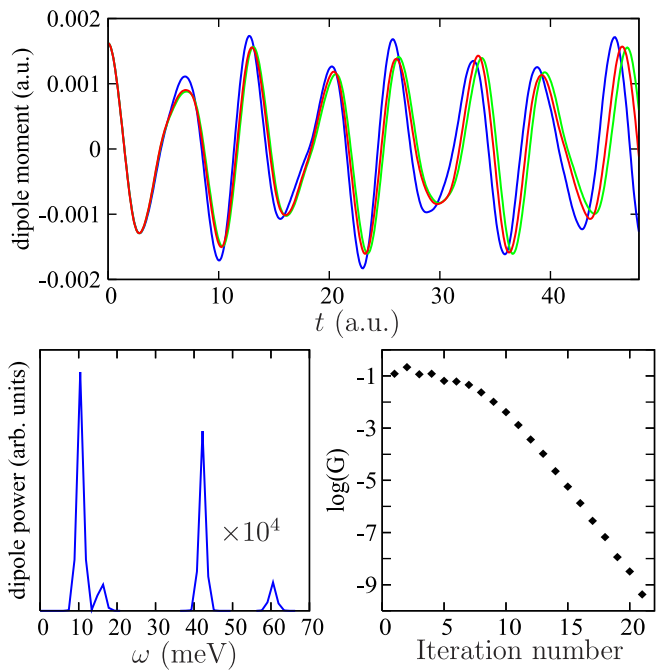

FIG. 1 (color online). Top: dipole moment of free chargedensity oscillations in a quantum well with two occupied subbands [blue, red, green (dark, medium, light gray): TDOEP, AOEP, TDKLI]. Bottom left: TDOEP dipole power spectrum. Bottom right: convergence index $G$ (see text).

$10.2 \mathrm{meV}$ in AOEP and TDKLI; $\omega_{23}, \omega_{14}$, and $\omega_{25}$ are at $16.0,42$, and $61 \mathrm{meV}$ in all three methods.

We find that the exact-exchange TDOEP does not cause any dissipation, similar to the high-frequency limit of TDCDFT [7,8]; the nonadiabatic XC contribution is thus purely elastic, i.e., phase shifted by $\pi$ with respect to the adiabatic part [21]. This is consistent with the observed behavior of the TDOEP versus AOEP, where the memory leads to a small frequency renormalization (blueshift) of the dominant $\omega_{12}$ plasmon.

Nonadiabatic effects play a pronounced role at high frequencies, when the system rapidly undergoes large deformations. The crossover from the low- to the highfrequency region occurs around the average plasma frequency of the system [21]. To explore different dynamic regimes, we now consider charge-density oscillations driven by $V_{\mathrm{dr}}(z, t)=e \mathcal{E} z f(t) \sin (\omega t)$, with electric field amplitude $\mathcal{E}$, intensity $I \sim \mathcal{E}^{2}$, and frequency $\omega$. The envelope $f(t)$ is switched on at $t_{0}=0$ over a 1 -cycle linear ramp and then kept constant.

Figures 2-5 show $d(t)$ for $\omega=11.2,20,40$, and $50 \mathrm{meV}$ and intensities $10,20,40$, and $1000 \mathrm{~W} / \mathrm{cm}^{2}$, respectively, probing the dynamics close to $\omega_{12}, \omega_{23}$, $\omega_{14}$, and between $\omega_{14}$ and $\omega_{25}$. As expected, the dipole response is largest at $11.2 \mathrm{meV}$ since we are close to the dominating plasmon. At $50 \mathrm{meV}$, far away from any resonance, we need a much larger intensity. Again, the three methods give comparable results for $d(t)$. Figures 2 and 3 show that AOEP falls in between TDOEP and TDKLI, but remains closer to TDKLI. For $40 \mathrm{meV}$ there are more pronounced differences, probably due to crosstalk of several plasmon resonances slightly off tune. Interestingly, at $50 \mathrm{meV}$, the results for $d(t)$ are very close. We found a similar behavior at low frequencies well below $\omega_{12}$. This

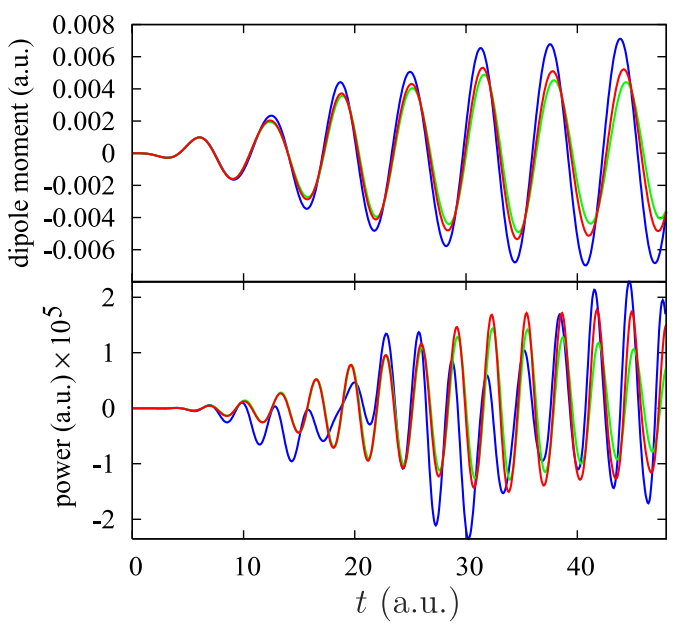

FIG. 2 (color online). Top: Dipole oscillations driven by an external field with frequency $\omega=11.2 \mathrm{meV}$ and intensity $I=$ $10 \mathrm{~W} / \mathrm{cm}^{2}$. Bottom: XC power [Eq. (4)]. Blue, red, green (dark, medium, light gray): TDOEP, AOEP, TDKLI.

suggests that at intermediate frequencies (not too far from the lowest intersubband plasmons), memory effects play a significant role only in the vicinity of resonances.

For a more detailed analysis, especially of the phase shifts of $V_{x \sigma}$, it is useful to consider the XC power:

$$
P(t)=\int d z j(z, t) \nabla_{z}\left[V_{x \sigma}(z, t)-V_{x \sigma}^{0}(z)\right],
$$

where $j(z, t)=\sum_{k}^{\text {occ }} \Im\left[\phi_{k \sigma}^{*}(z, t) \nabla_{z} \phi_{k \sigma}(z, t)\right]\left(\epsilon_{F}-\epsilon_{k \sigma}\right) / \pi$ is the current density. Since there is no dissipation, $P(t)$ is zero on average, but fluctuates at least twice as fast as $d(t)$ (it contains a product of two oscillating quantities).

The differences between TDOEP versus AOEP and TDKLI are much more apparent in $P(t)$ (bottom panels of Figs. 2-5), in particular, for higher frequencies. At $20 \mathrm{meV} V_{x \sigma}$ picks up a significant phase shift compared to AOEP and TDKLI, which themselves are completely in

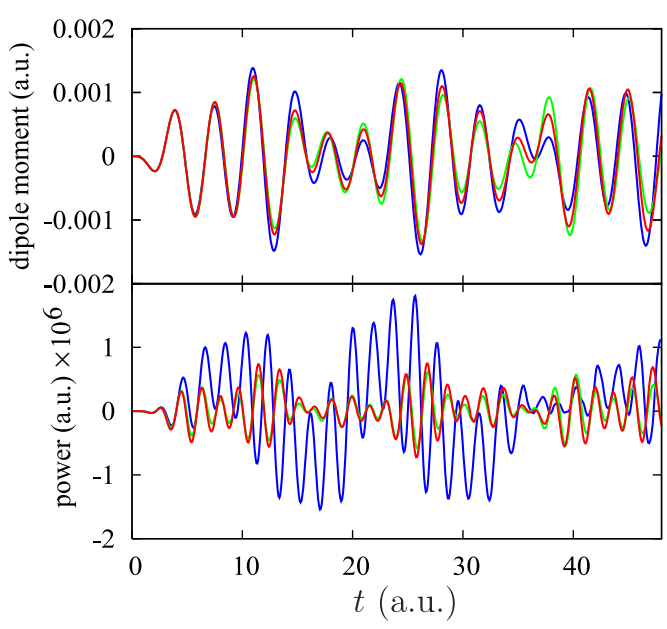

FIG. 3 (color online). Same as Fig. 2, with $\omega=20 \mathrm{meV}$ and $I=20 \mathrm{~W} / \mathrm{cm}^{2}$. 


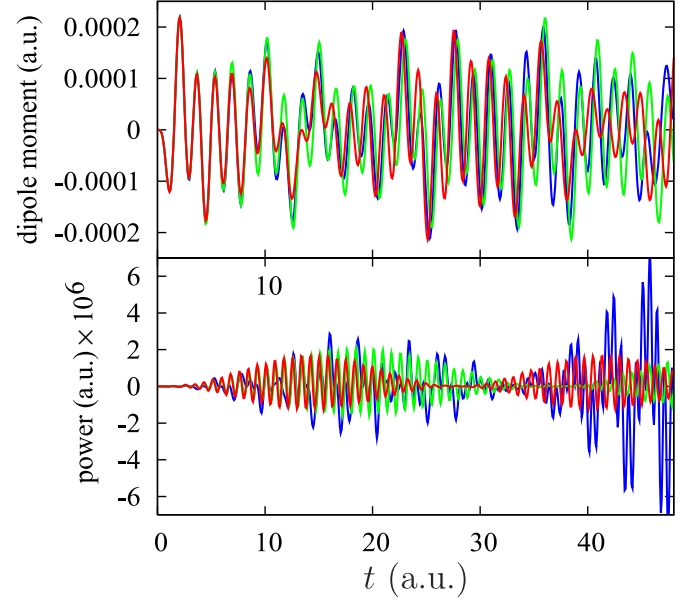

FIG. 4 (color online). Same as Fig. 2, with $\omega=40 \mathrm{meV}$ and $I=40 \mathrm{~W} / \mathrm{cm}^{2}$.

sync. This is a clear indication of memory-induced elasticity. The effect becomes even more pronounced at 40 and $50 \mathrm{meV}$. We also find that $P(t)$ exhibits characteristic beating patterns (in particular at $40 \mathrm{meV}$ ), which are due to the detuning between the driving field and the plasmon resonances. These patterns look similar in AOEP and TDKLI, but exhibit marked additional structures in TDOEP. Remarkably, as seen most clearly at $50 \mathrm{meV}$, these strong and rapid fluctuations of $V_{x \sigma}$ in TDOEP leave hardly any imprint on $d(t)$.

Conclusion. - We have developed numerical algorithms for the full TDOEP and its adiabatic approximation, the AOEP. The role of memory was analyzed for the collective electron dynamics in quantum wells. Away from resonances, AOEP and TDKLI closely agree (like ground-state OEP and KLI [14]) and are good approximations to the full TDOEP. Memory effects become more significant in the vicinity of intersubband resonances, resulting in additional elastic contributions to the dynamics.

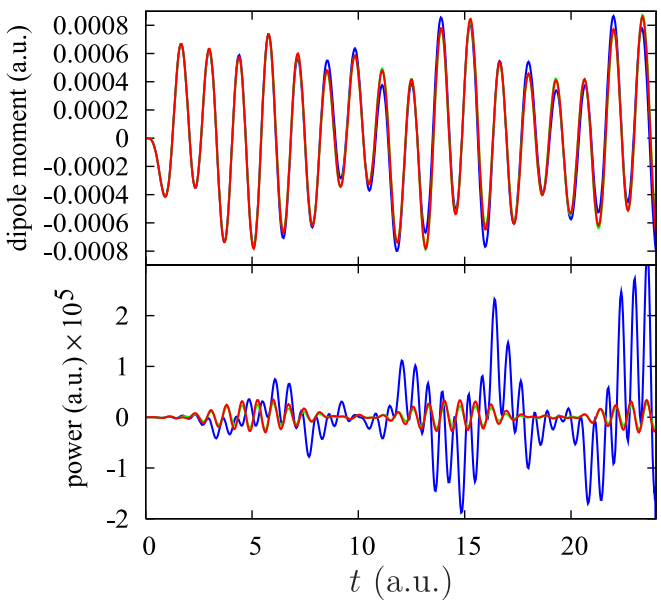

FIG. 5 (color online). Same as Fig. 2, with $\omega=50 \mathrm{meV}$ and $I=1 \mathrm{~kW} / \mathrm{cm}^{2}$.
Exact-exchange TDOEP has no memory at all in systems with only one occupied level [14], and our quantum well had only a small population of the second subband. Nonadiabatic effects should become more important for larger relative occupancies of upper levels, and in frequency regimes further above the lowest excitations.

Our analysis of the XC power shows that the full TDOEP has much richer temporal features than the adiabatic approximations, but this has relatively little impact on the electron dynamics itself. This suggests that for intrinsically nonadiabatic phenomena such as multiple excitations and dissipation one needs to go beyond exact exchange. Further exploration of orbital functionals thus remains an important task in TDDFT.

This work was supported by Research Corporation and by NSF Grant No. DMR-0553485. We thank Paul de Boeij for discussions and Sashi Satpathy for computer time.

*Present address: Department of Physics and Astronomy, Hunter College and CUNY, New York, NY 10021, USA.

[1] E. Runge and E. K. U. Gross, Phys. Rev. Lett. 52, 997 (1984).

[2] P. Elliott et al., arXiv:cond-mat/0703590.

[3] N. T. Maitra et al., J. Chem. Phys. 120, 5932 (2004).

[4] W. Hieringer and A. Goerling, Chem. Phys. Lett. 419, 557 (2006).

[5] G. Onida et al., Rev. Mod. Phys. 74, 601 (2002).

[6] M. Lein and S. Kümmel, Phys. Rev. Lett. 94, 143003 (2005).

[7] G. Vignale et al., Phys. Rev. Lett. 79, 4878 (1997).

[8] H. O. Wijewardane and C. A. Ullrich, Phys. Rev. Lett. 95, 086401 (2005).

[9] C. A. Ullrich and G. Vignale, Phys. Rev. Lett. 87, 037402 (2001).

[10] M. van Faassen et al., Phys. Rev. Lett. 88, 186401 (2002).

[11] C. A. Ullrich and K. Burke, J. Chem. Phys. 121, 28 (2004); C. A. Ullrich, J. Chem. Phys. 125, 234108 (2006).

[12] A. Goerling, J. Chem. Phys. 123, 062203 (2005).

[13] J.D. Talman and W.F. Shadwick, Phys. Rev. A 14, 36 (1976).

[14] J. B. Krieger et al., Phys. Rev. A 45, 101 (1992).

[15] C. A. Ullrich et al., Phys. Rev. Lett. 74, 872 (1995).

[16] R. van Leeuwen, Phys. Rev. Lett. 76, 3610 (1996); Phys. Rev. Lett. 80, 1280 (1998).

[17] A. Görling, Phys. Rev. A 57, 3433 (1998).

[18] Y.-H. Kim and A. Görling, Phys. Rev. Lett. 89, 096402 (2002).

[19] Y. Shigeta et al., Phys. Rev. A 73, 010502(R) (2006).

[20] M. Mundt and S. Kümmel, Phys. Rev. A 74, 022511 (2006).

[21] C. A. Ullrich and I. V. Tokatly, Phys. Rev. B 73, 235102 (2006).

[22] R. van Leeuwen and E. J. Baerends, Phys. Rev. A 49, 2421 (1994).

[23] F. A. Reboredo and C. R. Proetto, Phys. Rev. B 67, 115325 (2003). These authors treat quantum wells as open systems; we here consider fixed particle numbers.

[24] M. Mundt et al., Phys. Rev. A 75, 050501(R) (2007). 